\title{
Book Review: Collective Complexity out of Individual Simplicity
}

\author{
Alcherio Martinoli \\ Microsystems Laboratory, \\ Collective Robotics Group \\ California Institute of \\ Technology \\ Pasadena, CA 91125 \\ alcherio@micro.caltech.edu \\ http://www.micro.caltech.edu/ \\ research/CORO/index.htm
}

Swarm Intelligence: From Natural to Artificial Systems. Eric Bonabeau, Marco Dorigo, and Guy Theraulaz. 1999, Oxford University Press. 309 pages. \$29.95, paper.

The concept of Swarm Intelligence (SI) was first introduced by Gerardo Beni, Suzanne Hackwood, and Jing Wang in 1989 when they were investigating the properties of simulated, self-organizing agents in the framework of cellular robotic systems [1]. Eric Bonabeau, Marco Dorigo, and Guy Theraulaz extend the restrictive context of this early work to include "any attempt to design algorithms or distributed problem-solving devices inspired by the collective behavior of social insect colonies," such as ants, termites, bees, wasps, "and other animal societies." The abilities of such systems appear to transcend the abilities of the constituent individuals. In most biological cases studied so far, robust and capable high-level group behavior has been found to be mediated by nothing more than a small set of simple low-level interactions between individuals, and between individuals and the environment. The SI approach, therefore, emphasizes parallelism, distributedness, and exploitation of direct (agent-to-agent) or indirect (via the environment) local interactions among relatively simple agents.

The title, Swarm Intelligence: From Natural to Artificial Systems, summarizes the content and the structure of the book well: Each of the central chapters starts by presenting experimental results of one or several biological studies (foraging, division of labor, clustering and sorting, nest building, cooperative transportation), then describes a model for explaining these results, and moves on to discuss engineering outcomes in the form of algorithms or collective robotic systems that have or could have been inspired by the biological examples. One of the strengths of this monograph is undoubtedly this extensive use of models as a quantitative and abstract interface for the implementation of natural principles in artificial systems. Without an adequate level of description provided by models, it would be difficult, if not impossible, to understand the collective behavior of natural systems or to explore parametric ranges, which can be of interest for engineering purposes, though not necessarily useful in nature.

While the biological examples and their related modeling are well structured, the book tends to be less systematic in the myriad of engineering case studies that are associated with them. The global picture is far from being exhaustive and unitary: The book suffers from the current youth and rapidly developing nature of the SI field. The field currently lacks mature and sound methodologies to transfer biological mechanisms into useful engineering algorithms or to choose an adequate level of description for modeling. Bio-inspiration is a process fully dominated by the intuition and imagination of a few researchers. A full body of theory for designing and describing such distributed 
systems is still missing.

In the rest of this review, I will address separately the three main components of Swarm Intelligence: biological systems, algorithms, and robotic systems. Because my background and current research interests focus on robotics, I will devote more attention to the contributions of the book to this topic. I will then conclude the review with a few general remarks on the future of the SI field and the role of Swarm Intelligence in this context.

\section{Biological Systems}

As mentioned above, Swarm Intelligence introduces and utilizes models to fairly accurately explain the mechanisms underlying collective biological systems. In particular, the authors stress the role of two key ingredients of SI: self-organization and stigmergy. According to Bonabeau, Dorigo, and Theraulaz, theories of self-organization were "originally developed in the context of physics and chemistry to describe the emergence of macroscopic patterns out of processes and interactions defined at microscopic level" [9]. Jean-Louis Deneubourg, a major source of inspiration for this book, was one of the pioneers in the late 1970s in extending these theories to the field of social insects [3]. The concept of stigmergy, a form of indirect communication among teammates through the environment, was introduced in 1959 by Pier-Paul Grassé [7].

The extended treatment of these two major ingredients obscures other mechanisms that have been discovered in social insects and could play an important role, particularly for robotic implementations such as direct insect-to-insect communication and adaptation at the individual level (e.g., learning). Although these mechanisms have been less extensively studied from a quantitative point of view, their role and implications in the functioning of a colony of social insects could be fundamental (see, for instance, the work of Deborah Gordon, Jean-Louis Deneubourg, Mandyam Srinivasan, and Robert Page). The simplicity of the individual insects depicted in the book does not necessarily correspond to reality: Contacting rate regulation in ants [6], learning and individual memory in ants' foraging patterns [4], learning in bees' navigation [11], and task specialization [2] are just a few examples of intriguing mechanisms that are only partially or not at all mentioned.

Furthermore, in contrast with the SI definition mentioned above, Swarm Intelligence deals with social insect colonies, but not with other animal societies; collective movement phenomena in vertebrates, such as swarming, flocking, herding, and shoaling, are completely absent. Fish societies, for instance, can consist of thousands of individuals (one shoal of herring was reported to be 17 miles long) that can communicate in an indirect way by generating pressure waves. Although none of the authors have been actively working on such topics, such phenomena really should belong in a book entitled Swarm Intelligence.

\section{Algorithms}

The algorithmic sections of the book are those that, among the engineering applications reported, show the most sound results. I especially appreciated the comparison of SI-based algorithms with traditional ones using standard benchmarks, in particular in the chapter dealing with Ant Colony Optimization algorithms for static problems (e.g., Traveling Salesman Problem, Quadratic Assignment Problem) and dynamic problems (e.g., load balancing in telecommunication networks). I believe that the direction indicated in Swarm Intelligence for optimization is very promising and several other applications could benefit from swarm-based principles, particularly those that deal with problems characterized by intrinsic dynamic components. What is still missing, 
not only in Swarm Intelligence but also in the whole swarm-based algorithmic research field in general, are proofs of convergence and stability, methodologies for encoding the problem, and approaches for designing heuristic functions.

\section{Robotic Systems}

From an architecture point of view, there are three main advantages of the SI approach to (mobile) robot control. First, the resulting collective systems are scalable because the control architecture is exactly the same from a few units to thousands of units. Second, such systems are flexible because individual units can be dynamically added or removed without explicit reorganization by the operator. Third, these systems are robust, not only due to unit redundancy but also through minimalist unit design.

The sections dedicated to robotic experiments do not clearly outline these three advantages, and are among those that contribute the most to the heterogeneous, unstructured picture of the engineering applications arising from the monograph. On the one hand, this book mirrors the current situation in swarm robotics, in which each researcher is implementing his own control algorithms, often on a custom-developed hardware platform, and tackling tasks that are always slightly different from those of his colleagues. Under these constraints, it is very difficult to compare the efficiency of different robotic implementations on a given standard problem, such as was done for the Ant Colony Optimization algorithms. For instance, there does not (yet) exist a standard benchmark, such as the soccer tournament in small-group collective robotics, which addresses issues such as static and dynamic scalability as well as minimalism in the individual unit's design. On the other hand, perhaps because none of the authors is a roboticist (the originality of the robotic experiments seems to be "the only level the authors of this book can judge"), Swarm Intelligence does not contribute at all to demystifying the general impression of a perhaps too rapidly growing field, nor to convincing the general robotics community that SI is a novel and robust enough paradigm for controlling groups (or swarms) of robots.

The motivations of my criticism are threefold. First, the authors seem to associate the SI paradigm to the concept of a distributed control architecture and not to is aware of the work of other roboticists that developed efficient distributed architectures, which, for one reason or another, cannot be classified as SI-based. For instance, the ALLIANCE architecture proposed by Lynne Parker is distributed, fault-tolerant, and robust enough for controlling homogeneous and heterogeneous teams of robots [10]. However, it requires that each robot be aware of the other robots' current activities via wireless explicit communication in order to achieve cooperative team work, and is, therefore, well-suited for controlling small groups, but not swarms of robots (i.e., it is not scalable). Second, the authors seem to consider a group of robots more as a sort of collective embedded emulator of natural societies rather than as another type of system sensing and acting in the physics of the real world. It may be true that robotic experiments can help shed light on specific biological mechanisms that only real-world physical interactions can generate. For instance, in the special issue on stigmergy presented by this journal in 1999 [8], Owen Holland and Chris Melhuish reported results in annular sorting using real robots that matched observations in Leptothorax ants much more closely than those obtained eight years before by Jean-Louis Deneubourg using Monte-Carlo simulations [5]. However, these cases represent the exception rather than the rule. Designing and working with real robots is so time-consuming and inflexible in comparison to simulation that such an effort should at least benefit both fields, biology and robotics (e.g., in the terms of novel approaches for standard problems). Third, the authors do not adapt the SI metaphor to the current (mobile) robotic technology. A swarm of robots presents substantial dissimilarities to a natural swarm: On the one hand, sensing and grasping 


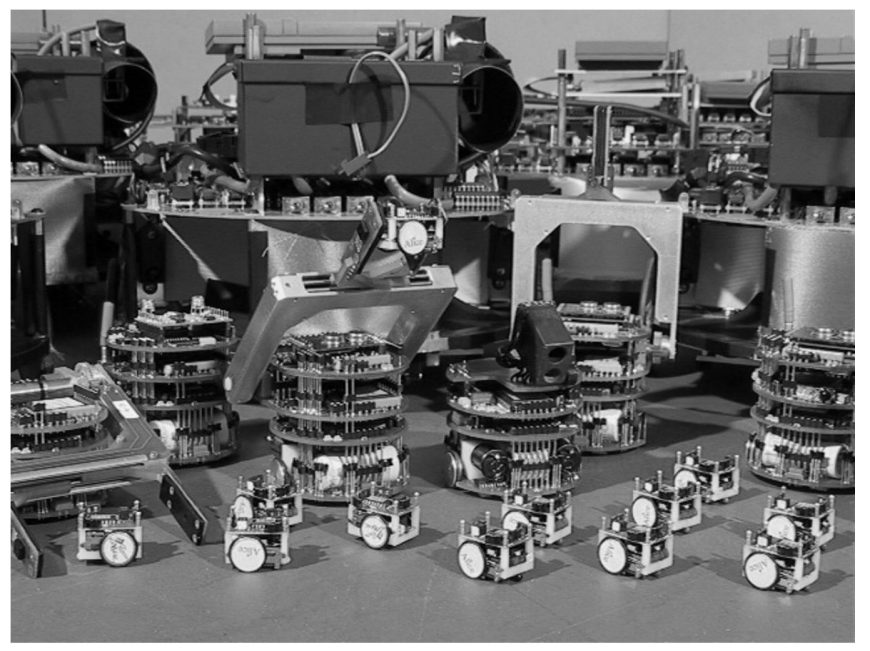

Figure I. The swarm of robots used in the Collective Robotics Group (CORO) at the California Institute of Technology, where the author works. Moorebots $(25 \mathrm{~cm}$ in diameter) were developed at University of West England, Bristol, in Alan Winfield's laboratory. Khepera $(5.5 \mathrm{~cm}$ in diameter) were developed at the Swiss Institute of Technology, Lausanne, in Jean-Daniel Nicoud's laboratory. Alices $(2 \mathrm{~cm}$ in length) were developed at the Swiss Institute of Technology, Lausanne, in Roland Siegwart's laboratory. Alice and Khepera are now commercialized by K-Team SA, Préverenges, Switzerland.

capabilities as well as mobility are much more developed in animals than robots; on the other hand, communication capabilities are much faster and long range in robots than in animals. We can therefore use all the possible available engineering techniques, such as explicit robot-to-robot communication or GPS technology, that could simplify the complexity of the individual while maintaining its autonomy, increasing the team efficiency, and still allowing the static or dynamic scalability of the architecture. For instance, explicit robot-to-robot communication would not become "a big issue when the number of robots increases" if simple signaling schemes based on local broadcast protocols are used. Thus, groups of robots collectively transporting heavy objects-a topic mentioned in the last chapter of the book-would be able to achieve a higher efficiency (maybe a superlinear one) than that obtained so far with strictly biomimetic principles.

\section{Conclusion}

In this review, I have presented my personal opinion about the book, which is clearly influenced by my engineering background. I will conclude these few notes with some more general considerations about the role this book plays and will play in the future of the SI field, in which I am also actively working.

I believe that Swarm Intelligence represents the first interdisciplinary attempt to define the SI field in a structured way and definitely contributes to legitimizing this young and developing field in a self-contained form. Bonabeau, Dorigo, and Theraulaz are to be congratulated in this regard and have undoubtedly succeeded in pursuing their main goal: "showing the promise of this approach and stimulating researchers to overcome some identified issues." However, a lot of work still has to be done to advance the maturity and credibility of the SI field, in particular by developing theory, designing methodologies, systematically assessing the validity of the approach for real- 
world problems, and, whenever possible, comparing its results with those of more classical techniques.

Finally, even though Swarm Intelligence is not a textbook, we at the Microsystems Laboratory (myself together with Rodney Goodman and Owen Holland) decided to follow the suggestion of the authors to use this monograph "to propagate our excitement to new generations of researchers." It has been a book enjoyed by both students and faculty alike, and I recommend it to you. The results of the first two iterations of the SI course at Caltech can be seen at http://www.micro.caltech.edu/Courses/EE141/. Enjoy!

\section{References}

1. Beni, G., \& Wang, J. (1989). Swarm Intelligence. In Proceedings of the Seventh Annual Meeting of the Robotics Society of Japan, Tokyo, Japan, (pp. 425-428).

2. Calderone, N. W., \& Page, R. E. (1996). Temporal polyethism and behavioural canalization in the honey bee. Apis Mellifera Animal Behavior, 51, 631-643.

3. Deneubourg, J.-L. (1977). Application de l'ordre par fluctuations à la description de certaines étapes de la construction du nid chez les termites. Insectes Sociaux, 24, 117-130.

4. Deneubourg, J.-L., Goss, S., Pasteels, J.-M., Fresneau, D., \& Lachaud, J.-P. (1987). Self-organization mechanisms in ant societies (II): Learning in foraging and division of labor. In J.-M. Pasteels and J.-L. Deneubourg (Eds.), From Individual to Collective Behavior in Social Insects (pp. 177-196). Experientia Supplementum, Vol. 54, Basel, Switzerland: Birkhäuser.

5. Deneubourg, J.-L, Goss, S., Franks, N., Sendova-Franks, A., Detrain, C., \& Chrétien, L. (1990, September). The dynamics of collective sorting: Robot-like ant and ant-like robot. In J.-A. Meyer and S. W. Wilson (Eds.), Proceedings of the First International Conference on Simulation of Adaptive Behavior: From Animals to Animats, Paris, France, (pp. 356-365).

6. Gordon, D. M., Paul, R. E., \& Thorpe, K. (1993). What is the function of encounter patterns in ant colonies? Animal Behavior, 45, 1083-1100.

7. Grassé, P.-P. (1959). La reconstruction du nid et les coordinations inter-individuelles chez Bellicositermes Natalensis et Cubitermes sp. La théorie de la stigmergie: essai d'interprétation du comportement des termites constructeurs. Insectes Sociaux, 6, 41-80.

8. Holland, O. E., \& Melhuish, C. (1999). Stigmergy, self-organization, and sorting in collective robotics. Artificial Life, 5, 173-202.

9. Nicolis, G., \& Prigogine, I. (1977). Self-organisation in non-equilibrium systems. New York: Wiley.

10. Parker, L. E. (1998). ALLIANCE: An architecture for fault tolerant multirobot cooperation. IEEE Transactions on Robotics and Automation, 14(2), 220-240.

11. Zhang, S. W., Bartsch, K., \& Srinivasan, M. V. (1996). Maze learning by honeybee. Neurobiology of Learning and Memory, 66(3), 267-282. 